\title{
Erratum zu: Überweisungserfolg in Psychotherapie bei Patienten mit Persönlichkeitsstörungen - Therapeutische Konsequenzen
}

Henriette Löffler-Stastka, Sophie Frantal, Elisabeth Jandl-Jager

\section{Erratum zu: Wien Klin Wochenschr}

DOI 10.1007/s00508-010-1353-x

Im Beitrag „Überweisungserfolg in Psychotherapie bei Patienten mit Persönlichkeitsstörungen - Therapeutische Konsequenzen“ von Henriette Löffler-Stastka, Sophie Frantal, Elisabeth Jandl-Jager [Wien Klin Wochenschr 122(5-6): 165-72, 2010] wurde Victor Blüml nicht als Co-Autor abgedruckt.

Wir bedauern den Irrtum und übermitteln hiermit die korrigierte Version:

Überweisungserfolg in Psychotherapie bei Patienten

mit Persönlichkeitsstörungen - Therapeutische Konsequenzen

Henriette Löffler-Stastka, Sophie Frantal, Victor Blüml, Elisabeth Jandl-Jager

Die Originalversion des Artikels finden Sie unter

DOI: $10.1007 / \mathrm{s} 00508-010-1353-\mathrm{x}$

Korrespondenz: Dr. med. Henriette Löffler-Stastka, Univ.-Klinik für Psychoanalyse und Psychotherapie, Medizinische Universität Wien,

Währinger Gürtel 18-20, 1090 Wien, Österreich,

E-Mail: henriette.loeffler-stastka@meduniwien.ac.at 